\title{
Factors influencing organizational adoption and implementation of clinical genetic services
}

\author{
Alison B. Hamilton, PhD, MPH ${ }^{1,2}$, Sabine Oishi, PhD, MSPH ${ }^{1}$, Elizabeth M. Yano, PhD, MSPH ${ }^{1,3}$, \\ Cynthia E. Gammage, BA 1 , Nell J. Marshall, DrPH ${ }^{1,3}$ and Maren T. Scheuner, MD, MPH ${ }^{1,4}$
}

Purpose: We sought to identify characteristics of genetic services that facilitate or hinder adoption.

Methods: We conducted semi-structured key informant interviews in five clinical specialties (primary care, medical oncology, neurology, cardiology, pathology/laboratory medicine) within 13 Veterans Administration facilities.

Results: Genetic services (defined as genetic testing and consultation) were not typically characterized by informants $(n=64)$ as advantageous for their facilities or their patients; compatible with organizational norms of low cost and high clinical impact; or applicable to patient populations or norms of clinical care. Furthermore, genetic services had not been systematically adopted in most facilities because of their complexity: knowledge of and expertise on genetic testing was limited, and organizational barriers to utilization of genetic services were formidable. The few facilities that had some success with implementation of genetic services had knowledgeable clinicians interested in developing services and organizational-level facilitators such as accessible genetic test-ordering processes.

Conclusion: Adoption and implementation of genetic services will require a multilevel effort that includes education of providers and administrators, opportunities for observing the benefits of genetic medicine, strategies for reducing the complexity of genomic medicine, expanded strategies for accessing genetics expertise and streamlining utilization, and resources dedicated to assessing the value of genetic information for the outcomes that matter to health-care organizations.

Genet Med advance online publication 15 August 2013

Key Words: clinical genetic services; diffusion of innovations; implementation

\section{INTRODUCTION}

Genomic information can enhance delivery of patient-centered care through individualized diagnosis, prognosis, risk assessment, and selection of treatment. It has been widely recognized, however, that uptake of genetic services is challenging, with a considerable gap between genomic discoveries and translation to genetic services. ${ }^{1,2}$ Furthermore, even when genomic applications have proven validity and utility, there is low adoption within health-care organizations. ${ }^{3}$ Barriers to the adoption of genetic services have been identified at multiple levels: healthcare systems lack structures and processes to facilitate genetic medicine practice; health professionals are not typically educated in genetics and have little time to incorporate genetic services into their practice; and guidelines for genetic testing, referral, and management are limited. ${ }^{4-8}$ Addressing barriers to implementation is complex, requiring a multilevel approach that targets patient, provider, organizational, and policy issues. ${ }^{5}$ To that end, it is critical to improve our understanding of these multilevel barriers so that implementation strategies may be developed and executed to improve the integration of genomic information into routine health-care delivery.

We conducted an exploratory study to identify characteristics of genetic services that may facilitate or hinder their adoption in a health-care organization. We investigated these characteristics across five different specialties in the Veterans Health Administration (VHA), the largest health-care system in the United States, providing care to more than 6 million veterans at 153 health-care facilities and their associated clinics across the country. As it is to many integrated healthcare systems in the United States, genetic medicine is relatively new to the VHA. The infrastructure is developing, with the establishment of a national Genomic Medicine Service in 2010 and the recent creation of a few clinical genetics programs based at VHA facilities in Houston, Los Angeles, and Boston. Widespread implementation has not occurred, so we characterize this study as a "pre-implementation" study, i.e., a "developmental evaluation" of existing conditions relevant to adoption and delivery of genetic services, as a precursor to full-fledged implementation efforts. ${ }^{9}$

\section{Theoretical model}

\section{MATERIALS AND METHODS}

Using diffusion of innovation theory, we assessed pre-implementation conditions by investigating how genetic services (the "innovation," defined at the outset of each interview as "genetic testing and consultation," consistent with the

\footnotetext{
${ }^{1}$ VA HSR\&D Center of Excellence for the Study of Healthcare Provider Behavior, VA Greater Los Angeles Healthcare System, Los Angeles, California, USA; ${ }^{2}$ Department of Psychiatry and Biobehavioral Sciences, David Geffen School of Medicine, University of California, Los Angeles, Los Angeles, California, USA; ${ }^{3}$ Department of Health Policy and Management, Jonathan and Karin Fielding School of Public Health, University of California, Los Angeles, Los Angeles, California, USA; ${ }^{4}$ Department of Medicine, David Geffen School of Medicine, University of California, Los Angeles, Los Angeles, California, USA. Correspondence: Alison B. Hamilton (alison.hamilton@va.gov)
} 
Institute of Medicine definition) ${ }^{10}$ have begun to diffuse into the health-care system and the attributes of the innovation that have fostered or hindered uptake. ${ }^{11,12}$ The theory posits that certain attributes of emergent practices or innovations influence the ease with which they may naturally diffuse into routine use, versus those that require active, targeted implementation strategies. The theory also provides a framework to predict the likelihood of implementation success. It postulates that an innovation, such as genetic services, will be successfully adopted if it is perceived to be advantageous (relative advantage); compatible with organizational and individual values, norms, and needs (compatibility); simple to implement (complexity); usable in preliminary fashion on a small scale (trialability); clearly beneficial (observability); and amenable to adaptation (reinvention). For example, if clinicians observe benefits to patients who receive genetic services, or if they are able to manage the complexity of genetic services utilization, the diffusion of innovation theory would predict that utilization of genetic services would be more likely. ${ }^{12}$ This article examines these attributes (relative advantage, compatibility, complexity, trialability, observability, and reinvention (Table 1) of genetic services, a complex, multifaceted innovation that is emergent in the US Department of Veterans Affairs (VA) health-care system.

\section{Sample}

To inform our understanding of multilevel barriers and facilitators to organizational adoption and implementation, we conducted interviews with leaders of five clinical specialties within the VHA: primary care, medical oncology, neurology, cardiology, and pathology and laboratory medicine. These specialties were selected because pilot work and our clinical experience suggested that they span the spectrum of demand for and utilization of genetic services. From pilot work, we expected that thematic saturation in interviews would be achieved with 10-15 "key informants" (i.e., individuals who are uniquely knowledgeable, reliable, and able to generalize about their cultural or organizational settings) ${ }^{13}$ per specialty. Therefore, we aimed to interview 50-75 key informants (10-15 per clinical specialty) located in 5 facilities within the United States. Using publically available information, we identified the clinical chief from each of the specialties at the five facilities. Service chiefs were invited by e-mail or telephone to participate as key informants in a 30- to 45-min semi-structured telephone interview about the adoption and delivery of genetic services at their facility. Due to low response rate at one original facility, two facilities were added to the initial sample, for a total of six facilities in the initial sampling frame. Subsequently, in a snowball sampling approach, informants in the initial sample were asked to identify additional, potentially knowledgeable interview candidates either within or outside of their facility, resulting in key informants from an additional seven facilities. ${ }^{14}$ The institutional review board at the VA Greater Los Angeles Healthcare System approved all study procedures.

\section{Data collection}

All interviews were conducted by the lead author (A.H.), supported by at least two secondary interviewers (M.S., S.O., and/or C.G.) using a semi-structured interview guide covering six general domains that addressed the following preimplementation organizational conditions: (i) existing genetic services and arrangements for providing genetic services; (ii) types, training, and roles of professionals who provide

Table 1 Diffusion of innovation in service organizations applied to genetic services

\begin{tabular}{|c|c|c|}
\hline Attribute & Content & Operational definition for thematic analysis \\
\hline \multirow[t]{2}{*}{$\begin{array}{l}\text { Relative } \\
\text { advantage }\end{array}$} & \multirow[t]{2}{*}{$\begin{array}{l}\text { Clear, unambiguous advantage of the innovation in terms of } \\
\text { effectiveness or cost-effectiveness }\end{array}$} & $\begin{array}{l}\text { - Knowledge/awareness of the intended uses of genetic services } \\
\text { (medical, reproductive, and personal decision making) and } \\
\text { indications for genetic testing (e.g., diagnostic, predictive, and } \\
\text { pharmacogenetic) }\end{array}$ \\
\hline & & $\begin{array}{l}\text { - Knowledge/awareness of genetic tests with proven clinical } \\
\text { validity and utility }\end{array}$ \\
\hline \multirow[t]{2}{*}{ Compatibility } & \multirow{2}{*}{$\begin{array}{l}\text { "Fit" between innovation and organizational values, norms, } \\
\text { and perceived needs; as well as providers' values, professional } \\
\text { norms, and ways of working }\end{array}$} & $\begin{array}{l}\text { - Local or system-wide policies regarding coverage for genetic } \\
\text { services }\end{array}$ \\
\hline & & - Providers' attitudes toward new technologies \\
\hline \multirow[t]{2}{*}{ Complexity } & \multirow[t]{2}{*}{ Innovations that are simpler to use will be easier to adopt } & - Perceived complexity of genetic tests \\
\hline & & $\begin{array}{l}\text { - Barriers to utilizing genetic services (e.g., genetic tests not } \\
\text { available in the laboratory test order menu) }\end{array}$ \\
\hline Trialability & $\begin{array}{l}\text { Ability to experiment with innovation on limited basis (e.g., } \\
\text { with space, time, and resources) }\end{array}$ & $\begin{array}{l}\text { - Experiences with requesting genetic services and receiving } \\
\text { results }\end{array}$ \\
\hline Observability & Benefits of innovation observable by intended adopters & $\begin{array}{l}\text { - Opportunities to observe utility or cost-effectiveness of genetic } \\
\text { services in the health-care setting }\end{array}$ \\
\hline Reinvention & $\begin{array}{l}\text { Adaptation, refinement, or other modifications to suit } \\
\text { adopter needs }\end{array}$ & $\begin{array}{l}\text { - Efforts to integrate some form of genetic services into routine } \\
\text { clinical care }\end{array}$ \\
\hline
\end{tabular}


services; (iii) procedures for ordering genetic tests; (iv) barriers to adoption and delivery of genetic services; (v) relative priority of genetic services in specialty areas; and (vi) factors necessary for further development of genetic services, including professional development activities. After providing our definition of genetic services (see above), we began by asking our key informants to talk about genetic tests ordered by themselves, colleagues in their specialty, or other providers at their VA, allowing for mention of any test with a genetic basis that could actually be ordered at their site. Later in the interview, we queried about the availability and type of genetic consultative services. Therefore, a wide range of possible tests and consultation arrangements was described and helped to characterize a previously unknown spectrum of genetic services and service arrangements in the VA. Interviewers debriefed after each interview. Interviews were conducted between December 2009 and February 2011. Interviews were digitally recorded and professionally transcribed verbatim.

\section{Data analysis}

The interview transcripts were reviewed and edited for accuracy and were then analyzed using ATLAS.ti (ATLAS.ti Scientific Software Development; www.atlasti.com). Each set of interviews was analyzed by specialty, followed by cross-specialty analysis within and across sites. Analysis was led by A.H. and also conducted by S.O. and C.G., with frequent feedback from the broader team. The initial code list was based on the interview guide, using top-level codes to broadly categorize responses to each interview question. After applying these broad codes, the content of each code (i.e., the segments of text to which the code was assigned) was examined for central themes, with additional inductive codes created for themes that were held in common across a substantial proportion (typically 50\%) of informants. As transcripts became available from each specialty, codes and subcodes were revisited and refined, if needed. For example, the original "barriers to adoption" code became too voluminous and multidimensional, so it was replaced by codes for each type of barrier that was frequently described (e.g., "barrier-cost of genetic tests"). A subset (at least 20\%) of each specialty-specific cluster was independently coded by A.H. and either S.O. or C.G. after code definitions were developed by the team. Intercoder reliability in the application of codes was established over time by comparing independent coding, identifying discrepancies, and discussing and resolving discrepancies with a team consensus process. Characteristics of the innovation were identified by clustering interviews (i.e., making document "families") with similar characteristics (e.g., oncology, cardiology) and comparing code content within each cluster of interviews (e.g., benefits/ advantages of genetic tests for oncology). In addition, A.H., S.O., and C.G. independently extracted quotations that illustrated each of the innovation attributes; they compared their findings, collaboratively selected illustrative quotations, and reviewed with the broader team to achieve consensus.

\section{RESULTS}

\section{Key informant characteristics}

Sixty-four key informants completed the interview: 16 in primary care; 13 cardiology; 10 oncology; 12 neurology; and 13 pathology (Table 2). Most of these key informants (70\%) were from the initial sampling frame (six facilities).

\begin{tabular}{|c|c|c|c|c|c|c|}
\hline Facility & Primary care & Cardiology & Medical oncology & Neurology & Pathology & Total \\
\hline \multicolumn{7}{|c|}{ Northeast } \\
\hline \multicolumn{7}{|l|}{ Midwest } \\
\hline 2 & 3 & & 1 & 1 & 3 & 8 \\
\hline 3 & & & & 1 & & 1 \\
\hline \multicolumn{7}{|c|}{ Southeast } \\
\hline 5 & 3 & 3 & 1 & 1 & 1 & 9 \\
\hline 6 & & 1 & 2 & 3 & & 6 \\
\hline 7 & & & & & 1 & 1 \\
\hline \multicolumn{7}{|c|}{ South central } \\
\hline 10 & 1 & 1 & 2 & 3 & 2 & 9 \\
\hline 11 & 3 & 3 & 1 & & & 7 \\
\hline \multicolumn{7}{|l|}{ West } \\
\hline 12 & 3 & 3 & 1 & 2 & 2 & 11 \\
\hline 13 & 3 & 1 & 2 & 1 & 1 & 8 \\
\hline Total & 16 & 13 & 10 & 12 & 13 & 64 \\
\hline
\end{tabular}


Characteristics of genetic services: a complex organizational innovation

Genetic services were consistently referred to by informants as a "new technology" or an "innovation," consistent with our $a$ priori characterization of these services as relatively new to the VHA overall. Although some informants described "hot spots" of activity related to genetic services, most were not aware of genetic services at their respective facilities, or they were aware of limited services (e.g., ability to order some genetic tests in the laboratory menu in the electronic health record) that were not being routinely utilized. Themes identified as relevant to genetic service attributes that may foster or hinder adoption are summarized in Table 3, with accompanying illustrative quotations, and described below.

\section{Relative advantage}

Mention of clearly advantageous genetic tests or consultation services tended to be specialty specific, with primarycare and cardiology informants citing very few advantages, and oncology, neurology, and pathology informants sometimes referencing specific tests and genetic services compatible with those specialties (see the section on "Compatibility" below). Many of the informants had difficulty even assessing advantage, citing limited access to information on available genetic tests, the utility of genetic tests, and the appropriate use of genetic consultative services. Many discussed advantages or disadvantages of some generally well-known tests (e.g., BRCA1/2 for hereditary breast-ovarian cancer syndrome), although they themselves may not have ordered them. Pathologists frequently questioned the cost-effectiveness of genetic tests, citing limited clinical utility in the face of high cost.

In general, informants from all specialties described ambiguous added value of genetic testing for predicting disease or treatment outcomes as compared with existing practice, particularly for common diseases. Some pointed out that advantage could be lessened to the point of disadvantage, and even danger, when providers misunderstand test results. With respect to whole-genome/whole-exome testing, informants indicated that although there is future promise, current applicability to clinical care is unclear. Furthermore, while some informants emphasized the importance of having a knowledgeable professional to interpret test results, perceived low volumes of applicable cases contributed to a general sense that hiring such professionals would not be cost-effective.

\section{Compatibility}

The organizational value consistently raised across specialties was clinical utility, with many describing the VHA as "a utilitarian system." Genetic tests that would most directly inform clinical decision making, diagnosis, and prognosis tended to be seen as most compatible with overall organizational standards, or with specialty-specific norms. For example, in neurology, value was associated with genetic tests that could provide diagnostic certainty and prognostic clarity, particularly for rare, potentially devastating diseases, even if clinical management would not change. Contributions of test results to quality of life and counseling for lifestyle decision making and family-risk considerations were mentioned as valuable in neurology.

Testing that could provide specific guidance for chemotherapeutic choices and counseling for difficult decisions around personal cancer risk, as well as cancer risk for family members, was described as valuable in oncology. Applicability of genetic testing to cardiology practice was uniformly described as low, and there was no use of consultative services reported. In primary care, where the occasional rare genetic disease would be difficult to recognize, and the added value of genetic testing was ambiguous (see above), the "fit" with professional norms was not portrayed as compatible, except for the occasional usefulness of factor V Leiden testing and testing for hemochromatosis.

Access to a VA-based geneticist was reported at only 1 of the 13 sites and was described as compatible with "ways of working" when deciding whether to order genetic tests and what to do with the results. None of the informants reported having genetic counselors on site. However, many described access to genetic consultative services through arrangements with nonVA organizations, particularly academic medical centers.

\section{Complexity}

Informants described nearly every aspect of using genetic services as fraught with complexity for their specialties and their facilities. The knowledge base required for identifying patients with indications for genetic tests, meaningfully collecting and applying family history information, and selecting appropriate tests for a given case was described as vast. Interpretation of genetic test results was also viewed as having complex dimensions with implications for family members that many clinicians were not trained to consider. Complex logistic "hoop jumping" in genetic test ordering and approval processes were frequently described as frustrating barriers to testing. Navigating the leadership hierarchy to adopt even approved pathology laboratory practices was also described as a complicated process.

\section{Trialability}

Due to the limited access to clinical genetic consultative services and the limited ability to order genetic tests using the electronic health record, most informants had no first-hand experiences with genetic services. Experimentation with tests and off-site consultation was largely self-motivated and limited by the complexities outlined above; however, at the site that had recently implemented on-site genetic consultative services, an oncology informant reported using the service and appreciating improvements in ease of test ordering (through the geneticist), as well as the welcome solution to the problem of counseling patients about results with complex issues relating to disease risk and options for management. 


\section{Observability}

Overall, observability of the benefits of genetic services was seldom reported, again likely due to limited access and utilization. A small number of informants described learning of benefits through observation of use by a colleague in another specialty, or by experiences that arose in their work at other organizations, such as university clinics. Pathologists occasionally described difficulty implementing genetic testing in the laboratory until administrative leaders observed cost savings related to the use of such testing in the population served.

\section{Reinvention}

Informants did not report opportunities to make their own modifications to test ordering or consult request processes to suit their specialty-specific needs. When asked what modifications might prove helpful, informants suggested possible adaptations to the electronic health record, such as genetic test menus; checklists with criteria for ordering tests; embedded links to relevant guidelines; and automated support for collecting family histories. At one facility, local adopters had successfully created a neurogenetics clinic, but such sophisticated adaptations to local environments were rare.

\section{DISCUSSION}

We found that the characteristics of genetic services as an "innovation" are not compatible with uptake into the healthcare organization by simple diffusion; instead, adoption of clinical genetic services will require development of targeted organizational supports to strengthen the likelihood of adoption and implementation. Informant characterizations supported our a priori assumption that the VHA is, at best, in the early stages of adoption of genetic services. Informants reported a general lack of familiarity with and activity around genetic services in their facilities, with only a few facilities where knowledgeable clinicians had taken a particular interest in developing these services. For the most part, adoption of genetic services was in the "let it happen" mode, meaning that incorporation of these services into organizational practice was typically unpredictable, uncertain, emergent, adaptive, and self-organizing, occurring via mechanisms that were rarely facilitated, i.e., the services occurred by "osmosis," as one informant stated. ${ }^{12}$

Informants' perspectives on clinical genetic services suggest that considerable work is needed to improve knowledge and provide opportunities for organizations to experience the benefits of this innovation. Overall, relative advantage of genetic services was not compelling, with many informants indicating that evidence of clinical utility and guidelines for genetic tests were lacking, leading to obstacles in their use. This issue of evidence has been highlighted in recent commentaries. For example, Evans and Khoury ${ }^{15}$ suggest that accrual of evidence is critical to implementation and that "premature implementation," i.e., implementation without sufficient evidence of superior outcomes, can "carry a high price tag in terms of patient suffering and cost."
Informants indicated that benefits did not outweigh the costs of genetic services. Organizational values, in particular, were described as incompatible with the notion of using expensive tests for little clinical gain, and there was infrequent consideration of gains beyond clinical management of patients (e.g., gains in quality of life or related to reproductive decision making). Further compounding the challenging context for adoption was the commonly shared viewpoint that genetics as a field is highly complex and that the expertise needed to appropriately use genetic information is not typically available. Clearly, efforts to simplify the implementation of genetic services are needed to mitigate their complexity and clarify the applicability of specific tests and consultation practices to specific clinical contexts. Finally, because few informants' facilities had actually developed genetic services through a multilevel approach that, for example, limited the "hoop jumping" for ordering tests, the trials (trialability), observations (observability), and reinventions that might have contributed to knowledge and experience of previously unappreciated advantages and clinical compatibility were limited; only the few who had successfully used genetic services could speak to these characteristics.

Our findings support the need for structured and targeted implementation strategies for genetic services, rather than passive diffusion. The VHA is well positioned to achieve successful implementation of genetic services given the investment in the Million Veterans Program (a medical database designed to study how genes affect health; http://www.research. va.gov/mvp/) and the recent creation of the national Genomic Medicine Service, which is developing organizational structures and processes to improve delivery and outcomes relating to genetic services. Informed by the seven steps (noted in italics) for successful diffusion of innovations, we propose the following implementation strategies for the VHA's national Genomic Medicine Service: support technology assessment to inform evidence-based guidance and policy for genomic applications (find sound solutions); act as the hub to support and unify the clinical genetic programs that exist throughout the system (find and support innovators, invest in early adopters), and provide genetic counseling services via telehealth where such expertise is lacking (lead by example); convene meetings, perhaps virtually, of the genetic service providers in the VHA and highlight accomplishments to clinical and administrative leadership (make early adopter activity visible); and foster development of educational materials and clinical tools in the electronic health record systems at each VHA facility, creating opportunities for genetic service utilization (create slack for change) with the ability for local adaptation (trust and enable reinvention). ${ }^{16}$ In a recent review paper, Manolio et al. ${ }^{3}$ provide many specific suggestions for solutions to implementation challenges that are compatible with those suggested here.

Our study has a number of strengths and limitations that deserve mention. First, our findings are exploratory and not generalizable to the VHA or to other health-care organizations. Second, we designed our investigation to view implementation through the lens of five specialties with varying 


\section{Table 3 Characteristics of genetic services as reported by key informants}

Themes: perceived characteristics of genetic services

Relative advantage

Advantage of genetic tests over traditional practices is ambiguous for predicting disease or treatment outcomes, particularly for common diseases

Cost-effectiveness of expensive genetic tests ambiguous in adding value to clinical decision making

Advantage of genetic tests ambiguous and even disadvantageous in the hands of providers who may not have training to correctly interpret results

Advantage of on-site versus outside referrals to genetic professionals low or unclear because of perceived low volume of relevant cases

\section{Compatibility}

Tests that directly inform clinical decision making (i.e., diagnosis, prognosis, and treatment) are most compatible with organizational standards across specialties

Genetic testing fit professional norms in neurology, particularly for providing diagnostic information to patients about rare, potentially devastating diseases

Contributions of test results to quality of life and counseling for lifestyle decision making and family risk fit values in neurology

Testing for specific guidance in chemotherapeutic choices fit professional norms in oncology

Low applicability of genetic testing to cardiology practice

Limited fit in primary care, where rare genetic diseases are hard to identify, and the added value of genetic testing for common diseases is perceived as ambiguous (see above)

\section{Illustrative quotes}

"So far, the genetic testing doesn't do any better than standard risk factors in predicting vascular disease... diabetes, hypertension... putting those together and saying a person might have a stroke is about as accurate as any genetic test so far." (Neurology)

"If the situation is, "the patient has this genotype then don't use clopidogrel," that would be useful. If it's, "the patient has this type of genotype then use clopidogrel cautiously," that's really ambiguous... and it isn't really helpful to the provider." (Primary care)

"With late-onset Alzheimer's... if we get the genetic test, maybe it will show I have a $50 \%$ chance of getting it...As people grow older, near $25 \%$ get it anyway. So how much are you willing to pay for the difference between 25 and 50 or even 90[\%], because you stand a fair chance of getting it [regardless]?" (Neurology)

"Suppose, for example, a patient has a reason to have iron storage at a higher level in the body and they ask for a test to see if he has hereditary iron storage disease. Now if the guy has a known disease that causes that, there's no point in doing a genetic test. These kinds of tests... each one costs...\$1,200, $\$ 1,500$ for something that we know is going to be there anyway." (Pathology)

"The number of times that people will come referred to me and who have been told that, "You have Alzheimer's disease because you have this gene," it's a very regular circumstance... It's really opening up a very dangerous precedent." (Neurology)

"How would a genetic counselor fare here [at the VA] —-the more classical model of a genetic counselor? I suspect that it would take a long time for a genetic counselor to build up a clinical census. It might occur over time as there's greater knowledge on the part of providers that such an option exists, but I suspect it would take a long time and I don't know that there would ever be such a census as to guarantee the sustainability of such a clinic." (Primary care)

"I get frequent solicitations for cardiomyopathy genetic testing, but it's not clear to me how that makes my patient live longer or feel better." (Cardiology)

"The individual who is requesting the test ought to have a justification as to in what way that test is going to change management, or diagnosis, or quality of life, or prognosis, or something of that sort." (Neurology)

"You have a case of motor neuron disease.... One of the potential choices is that this is Kennedy disease.... The prognosis for this patient [changes] suddenly from... a death sentence in two, three years or less [if amyotrophic lateral sclerosis] to the natural history [of] twenty, thirty years with almost normal life expectancy and no disability. So even though we're not changing the particular treatment ... from a prognostic standpoint, it has a huge impact on quality of life." (Neurology)

"You can't ignore the issue of genetic counseling and risks to other family members; that's a very important part of the diagnosis. It's part of treating the patient; it's part of taking care of your patient." (Neurology)

"The tumor-specific [tests] basically guide therapy, so certain types of cancer treatments work in certain types of tumors and not in others.... So that's pretty much part and parcel of our decision-making process." (Oncology)

"At this point, it's more in the realm of arrhythmia specialists where the channelopathies, for example... have been described as being genetically based and atherosclerosis is to some extent... and that's the dominant thing that we deal with, but it's really so highly variable and so many genes involved that I don't think that our bread-and-butter disease is going to be the next one to be a big player." (Cardiology)

"Even [if there's] a new, easy test for a very rare condition, I think it's harder for primary care providers to incorporate, just because it's hard to remember something that you use on average once every two or three years." (Primary care) 
Table 3 Continued

\section{Themes: perceived characteristics of genetic services}

\section{Complexity}

Knowing when and which genetic tests to order is complex

Interpreting results of genetic tests is complex

Counseling patients regarding test results is complex

Implementing test ordering procedures at the organizational level is complex

\section{Illustrative quotes}

"We talk about genetic testing as if it's one thing, but it's obviously thousands of different individual tests and each one has its own indication." (Neurology)

"For lymphoma, there are well-documented data that a panel of few as seven cytogenetic abnormalities might be all you need to... identify the subgroup of patients... that have a more aggressive disease. That has never caught on as much as I thought five years ago and I think part of it is the complexity of interpretation." (Oncology)

"If [a patient] were BRCA1 positive, I could help them think it through, but it's not something where I know the risk numbers off the top of my head.... It would take a fair amount of homework on my part to get ready for that conversation, so to have someone else [on-site geneticist] who's very... fluent in it, is really helpful." (Oncology)

"If I have a patient with metastatic cancer who's dying of their disease, it doesn't really matter to them.... whether they have a genetic abnormality that led them to get the cancer... but it matters to their children and other family members... It's different than when you're ordering a test for someone who's at risk and then you're trying to help them put in context what to do about their risk." (Oncology)

"You have to get the know-how and then go through the big hurdle of educating the leadership upstairs.... The KRAS and the BRAF and microsatellite instability is a recent development.... They send us a directive about it so we develop the test and we say we need this piece of equipment. Now the administrator upstairs...they have to make a determination in their own mind whether they need to fund this one...so that is a big hurdle." (Pathology)

\section{Trialability}

No description of experimentation available to clinician adopters on limited trial basis

Geneticist on staff at one site allowed for successful experimentation with genetics consultation

Observability

Little observability available to clinician adopters but changed behavior when achieved

Little observability available to organization-level adopters but changed behavior when achieved

Reinvention

No description of adopter empowerment to modify processes to suit needs for genetic services

One neurologist innovated by creating an on-site neurogenetics clinic
"Having the expertise available is really valuable, to be able to just say okay, I'm concerned about this person...I have enough worry that I think it's worth sending them on. That makes my job easier... I can provide better care and do a better job of really ordering the right tests, because someone who is really an expert is making the recommendation." (Oncology)

"Even becoming aware of the availability of some of those [tests] is more by osmosis...I thought a patient might have hemochromatosis. I sent him to Gl and noticed that the consultant ordered the genetic testing that I didn't even know existed, so the next time I went ahead and did it." (Primary care)

"The literature was showing that [HCV genotyping was] going to be [in demand].... Before the clinician demanded it, I started the procedure here....After validation for one-and-a-half years, no one was ordering it. But when gradually they got to know it, now we have a huge number of the HCV genotyping requests." (Pathology)

"Two or three years ago, we had much more resistance [from the lab], and then once we demonstrated the clinical utility to the lab, they essentially capitulated." (Neurology)
"[The neurogenetics clinic] focused on neurogenetic issues and I always get the question, "Why would you ever want to do that in the VA? There isn't any neurogenetic disease in the VA." But of course, that's not true, and the most common things I see are Huntington disease and Charcot-Marie-Tooth disease and hereditary ataxia and muscular dystrophies...I've seen familial ALS, I have seen neurofibromatosis, I have seen tuberous sclerosis, I have seen familial Alzheimer disease." (Neurology)

ALS, amyotrophic lateral sclerosis; GI, gastroenterology; HCV, hepatitis C virus; VA, US Department of Veterans Affairs.

genetic services needs, which gave us a breadth of perceptions. However, we did not include administrators or others in the organizational hierarchy, nor did we include the patients themselves. It is likely that information drawn from nonclinicians and patients would provide different perspectives on the implementation and utility of genetic services. Finally, our qualitative approach allowed us to delve into key informants' perceptions and concerns. However, we cannot use these data to quantify the extent of our findings; instead, we have used these findings to inform the development of a Web-based, structured survey for administration to clinical chiefs at every VHA facility.

This study represents one of the first efforts to identify the characteristics of genetic services that hinder or facilitate their organizational adoption and implementation. However, other studies of innovation adoption have reported similar conclusions 
to ours, e.g., that the need for and potential compatibility of the innovation should be assessed in implementation planning ${ }^{14}$ and that evidence of effectiveness (in this case, evidence of clinical utility and cost-effectiveness) is a prerequisite for the initiation of innovation. ${ }^{17,18}$ Diffusion of innovation theory facilitated the interpretation of our findings and generated essential lessons that demonstrate gaps and inform strategies for improving adoption and implementation of genetic services. Understanding the attributes of genetic services-and how these attributes interrelatecan guide design of active, planned, multilevel implementation efforts ${ }^{5}$ with tailored application supports for health-care organizations seeking to adopt these services.

\section{ACKNOWLEDGMENTS}

This study was funded by the VA Health Services Research \& Development (HSR\&D) Service (IBD 09-101); pilot work conducted through the HSR\&D-funded Health Services Genomics Pilot Program (DNA 08-128) also informed this paper. At the time of the study, A.B.H. was an investigator with the Implementation Research Institute (IRI), at the George Warren Brown School of Social Work, Washington University, St. Louis; supported through an award from the National Institute of Mental Health (R25 MH080916-01A2) and VA HSR\&D QUERI. E.M.Y.'s effort was supported by a VA HSR\&D Senior Research Career Scientist Award (RCS 05-195). This work was further supported by the VA Greater Los Angeles HSR\&D Center of Excellence (HFP 94-028). A.B.H. had full access to all of the data in the study and takes responsibility for the integrity of the data and the accuracy of the data analysis. All authors are employed by the Department of Veterans Affairs. A.B.H., E.M.Y., and M.T.S. receive research funding from the VA HSR\&D Service.

The views expressed within are solely those of the authors and do not necessarily represent the views of the Department of Veterans Affairs or the United States government. Portions of this paper were presented in poster form at the VA HSR\&D/QUERI National Conference, National Harbor, MD, July 2012.

\section{DISCLOSURE}

The authors declare no conflict of interest.

\section{REFERENCES}

1. Khoury MJ, Berg A, Coates R, Evans J, Teutsch SM, Bradley LA. The evidence dilemma in genomic medicine. Health Aff (Millwood) 2008;27: $1600-1611$.

2. Rogowski WH, Grosse SD, Khoury MJ. Challenges of translating genetic tests into clinical and public health practice. Nat Rev Genet 2009;10: 489-495.

3. Manolio TA, Chisholm RL, Ozenberger B, et al. Implementing genomic medicine in the clinic: the future is here. Genet Med 2013;15(4):258-267.

4. Hedgecoe A. From resistance to usefulness: sociology and the clinical use of genetic tests. BioSocieties 2008;3:183-194.

5. Khoury MJ, Coates RJ, Fennell ML, et al. Multilevel research and the challenges of implementing genomic medicine. I Natl Cancer Inst Monographs 2012;2012:112-120.

6. Scheuner MT, Sieverding P, Shekelle PG. Delivery of genomic medicine for common chronic adult diseases: a systematic review. JAMA 2008;299:13201334.

7. Suther S, Goodson P. Barriers to the provision of genetic services by primary care physicians: a systematic review of the literature. Genet Med 2003;5: 70-76.

8. Taylor MR, Edwards JG, Ku L. Lost in transition: challenges in the expanding field of adult genetics. Am J Med Genet C Semin Med Genet 2006;142C: 294-303.

9. Stetler $C B$, Legro MW, Wallace $C M$, et al. The role of formative evaluation in implementation research and the QUERI experience. J Gen Intern Med 2006;21(suppl 2):S1-S8.

10. Institute of Medicine (US) Roundtable on Translating Genomic-Based Research for Health. Innovations in Service Delivery in the Age of Genomics: Workshop Summary. Genetic Service Delivery: The Current System and Its Strengths and Challenges. National Academies Press: Washington, D.C., 2009.

11. Rogers EM. Diffusion of Innovations, 5th edn. Free Press: New York, 2003.

12. Greenhalgh T, Robert G, Macfarlane F, Bate P, Kyriakidou O. Diffusion of innovations in service organizations: systematic review and recommendations. Milbank Q 2004;82:581-629.

13. Kumar N, Stern LW, Anderson JC. Conducting interorganizational research using key informants. Acad Manage J 1993;36:1633-1651.

14. Patton MQ. Qualitative Research \& Evaluation Methods, 3rd edn. Sage: Thousand Oaks, 2001

15. Evans JP, Khoury MJ. The arrival of genomic medicine to the clinic is only the beginning of the journey. Genet Med 2013;15(4):268-269.

16. Berwick DM. Disseminating innovations in health care. JAMA 2003;289:19691975.

17. Carlfjord S, Lindberg M, Bendtsen P, Nilsen P, Andersson A. Key factors influencing adoption of an innovation in primary health care: a qualitative study based on implementation theory. BMC Fam Pract 2010;11:60.

18. Barnett J, Vasileiou K, Djemil F, Brooks L, Young T. Understanding innovators' experiences of barriers and facilitators in implementation and diffusion of healthcare service innovations: a qualitative study. BMC Health Serv Res 2011;11:342. 